\title{
Presence of tertiary lymphoid structures determines the level of tumor-infiltrating lymphocytes in primary breast cancer and metastasis
}

\author{
Miseon Lee ${ }^{1}$ Sun-Hee Heo ${ }^{1,2} \cdot$ In Hye Song $\mathbb{D}^{1} \cdot$ Hajar Rajayi $^{1,2} \cdot$ Hye Seon Park ${ }^{1,2} \cdot$ In Ah Park ${ }^{1}$ Young-Ae Kim ${ }^{1,2}$. \\ Heejae Lee ${ }^{1,2} \cdot$ Gyungyub Gong $^{1} \cdot$ Hee Jin Lee $\mathbb{I}^{1}$
}

Received: 14 May 2018 / Revised: 16 July 2018 / Accepted: 21 July 2018 / Published online: 28 August 2018

(c) United States \& Canadian Academy of Pathology 2018

\begin{abstract}
The level of tumor-infiltrating lymphocytes and presence of tertiary lymphoid structures are significant prognostic and predictive factors in primary breast cancer. However, the understanding about differences in tumor-infiltrating lymphocytes and tertiary lymphoid structures at various metastatic sites or between primary breast tumors and metastatic sites is limited. A total of 335 cases of metastatic breast cancer from four metastatic sites (lung, liver, brain, and ovary) were included. We analyzed the percentages of tumor-infiltrating lymphocytes and presence of tertiary lymphoid structures in the primary and metastatic sites. The mean level of tumor-infiltrating lymphocytes in the lung metastases was higher than in the liver, brain, ovary, and matched primary tumors, while metastatic tumors of the liver and brain showed lower levels of tumor-infiltrating lymphocytes than primary tumors. Tertiary lymphoid structures were only found in the lung and liver, and in cases of brain metastases the change of tertiary lymphoid structures from present to absent significantly affected the level of tumorinfiltrating lymphocytes in metastases compared with that in matched primary tumors. Patients with a lower histological grade, hormone receptor positivity in primary tumors and metastases, a lower level of tumor-infiltrating lymphocytes and absence of tertiary lymphoid structures in primary tumors, a higher level of tumor-infiltrating lymphocytes and presence of tertiary lymphoid structures in metastases, and lung metastases showed significantly better overall survival. Our results showed that metastatic breast tumors in the lung had more tumor-infiltrating lymphocytes than did tumors at other sites and matched primary tumors. In addition, the presence of tertiary lymphoid structures in metastatic sites is a critical factor for the level of tumor-infiltrating lymphocytes.
\end{abstract}

\section{Introduction}

The level of tumor-infiltrating lymphocytes and presence of tertiary lymphoid structures are significant prognostic and

These authors contributed equally: Miseon Lee, Sun-Hee Heo

Gyungyub Gong

gygong@amc.seoul.kr

$\triangle$ Hee Jin Lee

backlila@gmail.com

1 Department of Pathology, Asan Medical Center, University of Ulsan College of Medicine, Seoul, Korea

2 Asan Center for Cancer Genome Discovery, Asan Institute for Life Sciences, Asan Medical Center, University of Ulsan College of Medicine, Seoul, Korea predictive factors in primary breast cancer [1]. Our recent studies showed that the level of tumor-infiltrating lymphocytes was a significant prognostic factor for the disease-free survival of patients with human epidermal growth factor receptor 2 (HER2)-positive and triple-negative breast cancers. In addition, higher tumor-infiltrating lymphocytes and tertiary lymphoid structure levels could be important predictors of a pathological complete response in triplenegative breast cancer [2-4].

Metastatic cancers are distinguished from primary cancer by microenvironments, such as immune-suppressive stroma. Some studies have evaluated tumor-infiltrating lymphocytes in lung metastasis from colorectal and renal cell carcinoma and in cutaneous metastases from melanoma $[5,6]$. However, our understanding of the differences in tumor-infiltrating lymphocytes and tertiary lymphoid structures at different metastatic sites or between primary breast tumors and metastatic sites is limited. 
Cancer immune microenvironments can be evaluated by various methods, such as the assessment of tumorinfiltrating lymphocytes on hematoxylin and eosin-stained slides, immune cell profiling using immunohistochemistry and flow/mass cytometry, and immune gene expression analysis from bulk tissue or dissociated single cells [7-11]. In breast cancer, the robustness of assessing tumor-infiltrating lymphocytes on hematoxylin and eosinstained slides of primary tumors has been proven by several clinical studies [7, 8, 12-17]. Recently, evaluation methods for tumor-infiltrating lymphocytes in metastatic lesion have been published by the International ImmunoOncology Biomarkers Working Group [1]. These evaluation methods are similar to the primary breast cancer assessment that is recommended by the same group [7].

The presence of tertiary lymphoid structures has been shown to be prognostic and predictive markers for breast cancer, although methods for evaluating the extent of tertiary lymphoid structures in cancer microenvironments have not been well established [2-4, 17, 18]. However, the impact of the presence of tertiary lymphoid structures in breast cancer metastatic lesions has never been explored. To address the differences of tumor-infiltrating lymphocytes and tertiary lymphoid structures among different metastatic sites and between primary breast tumors and metastatic sites, we evaluated the level of tumor-infiltrating lymphocytes and presence of tertiary lymphoid structures in a large cohort of patients with metastatic breast cancer. We also analyzed the prognostic significance of tumor-infiltrating lymphocytes and tertiary lymphoid structures in metastatic lesions.

\section{Materials and methods}

\section{Patient material}

Three hundred and thirty-five patients with metastatic breast cancers in the lung, liver, brain, or ovary, with available hematoxylin and eosin-stained slides of metastatic sites (biopsy, $n=172$; excision, $n=163$ ), who were treated at the Asan Medical Center from January 2000 to March 2017, were included. Among the 335 cases, hematoxylin and eosin-stained slides of primary breast cancer were also available for evaluating tumor-infiltrating lymphocytes and tertiary lymphoid structures for 245 cases (biopsy, $n=37$; excision, $n=208$ ). Exemption from informed consent, after information de-identification, was approved by the Institutional Review Board of the Asan Medical Center (2015-0880). This study was performed in accordance with the Declaration of Helsinki.

\section{Histological evaluation}

The hematoxylin and eosin-stained slides were reviewed by two pathologists (HJL and ML). The histological type of primary breast cancer was assessed based on the 2012 World Health Organization classification, and the histological grade of breast cancer was evaluated according to the modified Bloom-Richardson grading system. Full section slides were evaluated for stromal tumor-infiltrating lymphocyte levels (the $\%$ of stroma occupied by mononuclear cells including lymphocytes and plasma cells), in 10\% increments (if less than $10 \%, 0,1,2$, or $5 \%$ level criteria were used) [1]. Cases including adjacent non-tumorous tissue were assessed for the presence of tertiary lymphoid structures (defined as lymphoid aggregation with high endothelial venules) in primary and metastatic sites.

\section{Immunohistochemical analysis}

Formalin-fixed, paraffin-embedded tissue sections of primary and metastatic sites were stained with an automatic immunohistochemical staining device (Benchmark XT, Ventana Medical Systems, Tucson, AZ, USA). Antibodies for the estrogen receptor (ER; 1:200, Leica Biosystems, Newcastle, UK), progesterone receptor (PR; 1:200, Leica Biosystems), and HER2 (1:8, Ventana Medical Systems) were used. ER and PR levels were considered positive if the Allred score was higher than 2 [19]. Hormone receptorpositive tumors were classified by positivity on ER and/or PR staining. HER2-overexpressing tumors were classified by immunohistochemistry scores $3+$ scores or positive gene amplification, by silver in situ hybridization [20].

\section{Gene expression analysis}

We investigated mRNA expression of CXCL12, CXCL13, $C C L 21$, and $L T B$ between different types of normal tissue. Gene expression data of 2696 samples from normal breast, lung, liver, brain, and ovary was obtained from the Genotype-Tissue Expression project (GTEx v7) RNA-Seq analysis [21]. We also analyzed mRNA expression (RNA seqV2 RSEM) of four genes in 3795 samples from 7 The Cancer Genome Atlas Provisional datasets, including breast invasive carcinoma, lung adenocarcinoma and squamous cell carcinoma, hepatocellular carcinoma, brain low-grade glioma, glioblastoma, and ovarian serous cystadenocarcinoma [22].

\section{Statistical analyses}

All statistical analyses were carried out using SPSS version 20 (SPSS, Chicago, IL) and GraphPad Prism 7 (GraphPad 
$\mathbf{A}$

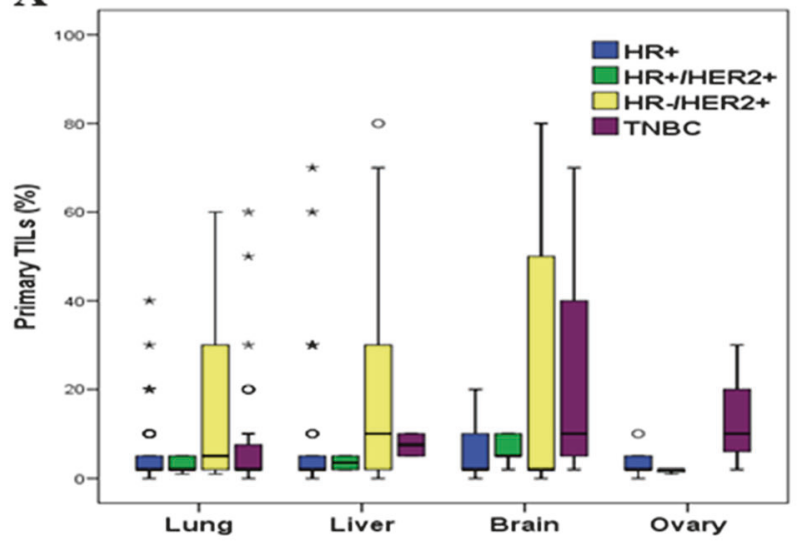

B

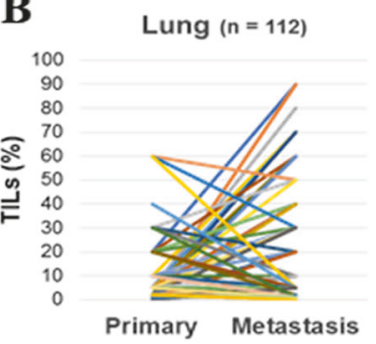

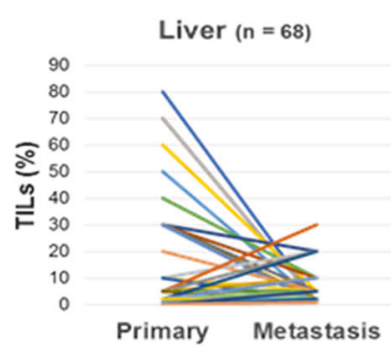

Primary Metastasis

Fig. 1 Comparison of tumor-infiltrating lymphocytes in primary tumors and metastases. a Comparison of tumor-infiltrating lymphocyte levels in primary tumors (left) and in metastatic sites (right), depending on breast cancer subtype. b Pair-wise comparisons of tumor-infiltrating

Software Inc., La Jolla, CA, USA). The Wilcoxon signedrank test, Mann-Whitney test, Spearman correlation, logrank test, and Cox proportional hazards regression model were used, as appropriate. All tests were two-sided and were performed with a significance level of $\alpha=0.05$.

\section{Results}

\section{Characteristics of primary and metastatic tumors and tumor-infiltrating lymphocytes in each metastatic site}

It is known that tumor-infiltrating lymphocyte levels in primary breast tumors differ with hormone receptor and HER2 statuses [23]. Therefore, we evaluated the tumorinfiltrating lymphocyte levels in each subtype, based on ER, PR, and HER2 expression in the metastatic lesions and corresponding primary breast tumors. Hormone receptornegative tumors showed higher tumor-infiltrating lymphocyte levels than hormone receptor-positive tumors in both primary breast cancer $(P=0.003)$ and metastatic tumors $(P=0.018$, Fig. 1a).

More than a third (38\%) of the primary tumors of lung metastases showed hormone receptor positivity and HER2
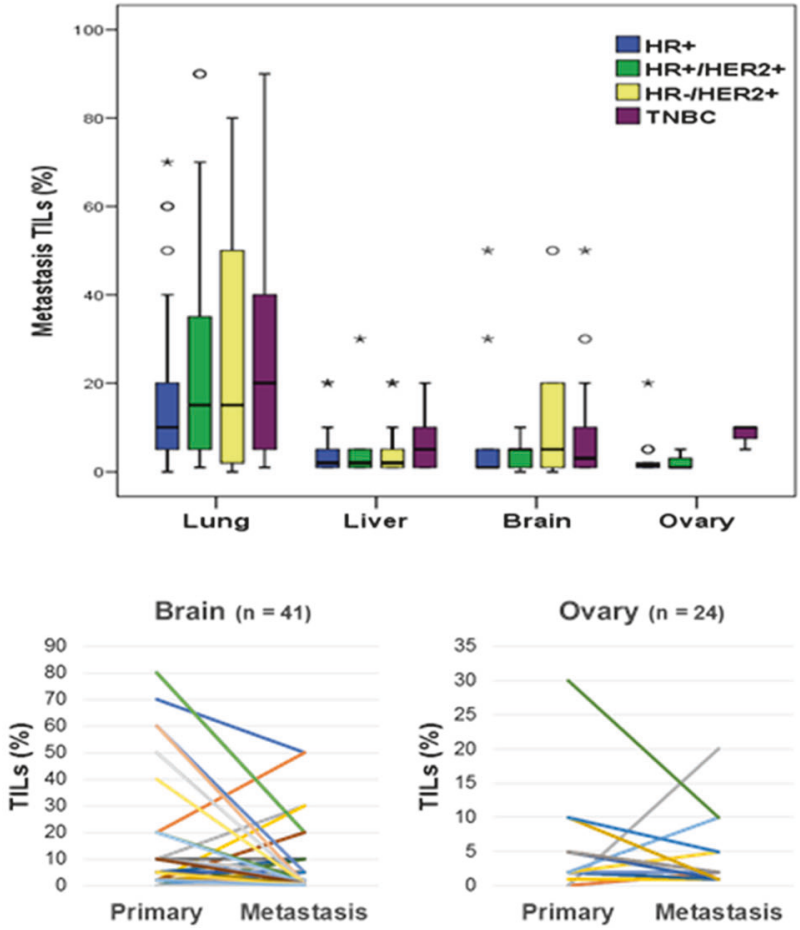

lymphocytes in primary tumors and matched metastases (lung, 112 pairs, $P<0.001$; liver, 68 pairs, $P=0.015$; brain, 41 pairs, $P=0.097$; ovary, 24 pairs, $P=0.413$ )

negativity (Table 1). The mean level of tumor-infiltrating lymphocytes in primary tumors of lung metastases was the third greatest among the four metastatic sites, and significantly lower than in the primary tumors of brain metastases $(P=0.036$, Table 1$)$. About half (48\%) of lung metastases from breast cancers also showed hormone receptor positivity and HER2 negativity (Table 2). Nevertheless, the mean level of tumor-infiltrating lymphocytes in lung metastases was the highest among all subtypes (Fig. 1a).

Primary tumors of liver metastasis and liver metastases from breast cancers had similar proportion of hormone receptor-positive and HER2-negative tumors to lung tumors (Tables 1 and 2). The percentage of tumors with tertiary lymphoid structures was lower in liver metastases than in primary tumors of liver metastasis.

Primary tumors of brain metastasis showed higher histological grade, hormone receptor negativity, and HER2 positivity (Table 1). The mean level of tumor-infiltrating lymphocytes was highest in the primary breast cancers in cases with brain metastases compared to other primary cancers with metastases to other sites (Fig. 1a). Brain metastases of breast cancers also showed higher hormone receptor negativity and HER2 positivity (Table 2). However, the mean level of tumor-infiltrating lymphocytes in 
Table 1 Characteristics of primary tumors according to tissue-obtained metastatic sites

\begin{tabular}{|c|c|c|c|c|}
\hline & \multicolumn{4}{|c|}{ Tissue-obtained metastatic sites } \\
\hline & Lung (\%) & Liver (\%) & Brain $(\%)$ & Ovary $(\%)$ \\
\hline \multicolumn{5}{|l|}{ Histological type } \\
\hline DCIS & $2(1)$ & $1(1)$ & $0(0)$ & $0(0)$ \\
\hline IDC & $158(99)$ & $94(97)$ & $52(98)$ & $23(92)$ \\
\hline ILC & $0(0)$ & $1(1)$ & $1(2)$ & $2(8)$ \\
\hline Mucinous carcinoma & $0(0)$ & $1(1)$ & $0(0)$ & $0(0)$ \\
\hline \multicolumn{5}{|l|}{ Histological grade } \\
\hline 1 & $4(3)$ & $1(1)$ & $0(0)$ & $0(0)$ \\
\hline 2 & $63(39)$ & 47 (49) & $14(26)$ & $18(72)$ \\
\hline 3 & $70(44)$ & $33(34)$ & $35(66)$ & $5(20)$ \\
\hline Unknown & $23(14)$ & $16(16)$ & $4(8)$ & $2(8)$ \\
\hline \multicolumn{5}{|l|}{ Hormone receptor } \\
\hline Negative & $57(36)$ & $29(30)$ & $28(53)$ & $2(8)$ \\
\hline Positive & $90(56)$ & $62(64)$ & $23(43)$ & $23(92)$ \\
\hline Unknown & $13(8)$ & $6(6)$ & $2(4)$ & $0(0)$ \\
\hline \multicolumn{5}{|l|}{ HER2 } \\
\hline Negative & $97(61)$ & $52(54)$ & $21(40)$ & $21(84)$ \\
\hline Positive & $41(26)$ & $31(32)$ & $29(55)$ & $4(16)$ \\
\hline Unknown & $22(14)$ & $14(14)$ & $3(5)$ & $0(0)$ \\
\hline \multicolumn{5}{|l|}{ Subtype } \\
\hline HR+/HER2- & $61(38)$ & $42(43)$ & $10(19)$ & $19(76)$ \\
\hline HR +/HER2+ & $22(14)$ & $12(12)$ & $13(25)$ & $4(16)$ \\
\hline HR-/HER2+ & $19(12)$ & $19(20)$ & $15(28)$ & $0(0)$ \\
\hline TN & $36(22)$ & $10(10)$ & $11(21)$ & $2(8)$ \\
\hline Unknown & $22(14)$ & $14(15)$ & $4(7)$ & $0(0)$ \\
\hline TIL $(\%$, median $)$ & 2 & 3.5 & 5 & 2 \\
\hline \multicolumn{5}{|l|}{ TLS } \\
\hline Absent & $52(32)$ & $42(43)$ & $18(34)$ & $21(84)$ \\
\hline Present & $60(38)$ & $26(27)$ & $23(43)$ & $3(12)$ \\
\hline Unknown & $48(30)$ & $29(30)$ & $12(23)$ & $1(4)$ \\
\hline
\end{tabular}

$D C I S$ ductal carcinoma in situ, $H R$ hormone receptor, $I D C$ invasive ductal carcinoma, ILC invasive lobular carcinoma, TIL tumorinfiltrating lymphocyte, $T L S$ tertiary lymphoid structure, $T N$ triplenegative

metastatic tumors was low, and even significantly lower than those of the lung $(P<0.001$, Fig. 1a).

Primary tumors of ovarian metastases were mostly hormone receptor positive and HER2 negative and showed the lowest mean tumor-infiltrating lymphocyte level (Table 1, Fig. 1a). The percentage of primary tumors with tertiary lymphoid structures was also the lowest in ovarian metastases (Table 1). Ovarian metastases still showed low tumorinfiltrating lymphocyte level (Table 2).

\section{Pair-wise comparison of tumor-infiltrating lymphocytes between primary and metastatic sites}

The level of tumor-infiltrating lymphocytes in primary tumors and metastatic lesions generally did not correlate with each other $(\rho=0.104, P=0.105)$. The mean level of tumor-infiltrating lymphocytes in metastatic tumors of the
Table 2 Characteristics of metastatic tumors

\begin{tabular}{|c|c|c|c|c|}
\hline & \multicolumn{4}{|c|}{ Tissue-obtained metastatic sites } \\
\hline & Lung (\%) & Liver $(\%)$ & Brain $(\%)$ & Ovary $(\%)$ \\
\hline $\begin{array}{l}\text { Age at diagnosis of first } \\
\text { distant metastasis (mean } \\
\pm \text { SD) }\end{array}$ & $50.9 \pm 10.8$ & $49.7 \pm 9.7$ & $48.4 \pm 8.7$ & $45.4 \pm 5.9$ \\
\hline \multicolumn{5}{|c|}{ Timing for diagnosis of first distant metastasis } \\
\hline de novo & $12(7)$ & $22(23)$ & $5(9)$ & $2(8)$ \\
\hline DMFI $\leq 6 \mathrm{mo}$ & $3(2)$ & $0(0)$ & $0(0)$ & $0(0)$ \\
\hline DMFI $>6 \mathrm{mo}, \leq 24 \mathrm{mo}$ & $23(14)$ & $23(24)$ & $26(49)$ & $6(24)$ \\
\hline DMFI > $24 \mathrm{mo}, \leq 60 \mathrm{mo}$ & $65(41)$ & $32(33)$ & $13(25)$ & $7(28)$ \\
\hline DMFI >60 mo & $57(36)$ & $20(20)$ & $9(17)$ & $10(40)$ \\
\hline \multicolumn{5}{|c|}{ First site of distant metastasis } \\
\hline Bone only & $2(1)$ & $4(4)$ & $3(6)$ & $5(20)$ \\
\hline Brain only & $0(0)$ & $1(1)$ & $39(73)$ & $1(4)$ \\
\hline Visceral only & $143(89)$ & $68(70)$ & $8(15)$ & $13(52)$ \\
\hline Visceral and bone & $11(7)$ & $22(23)$ & $1(2)$ & $6(24)$ \\
\hline Visceral and brain & $2(1)$ & $1(1)$ & $1(2)$ & $0(0)$ \\
\hline Other & $2(1)$ & $1(1)$ & $1(2)$ & $0(0)$ \\
\hline \multicolumn{5}{|c|}{ Number of metastatic masses during follow-up } \\
\hline Single & $30(19)$ & $7(7)$ & $6(11)$ & $0(0)$ \\
\hline Multiple & $130(81)$ & $90(93)$ & $47(89)$ & $25(100)$ \\
\hline \multicolumn{5}{|c|}{ Procedure for tissue obtaining } \\
\hline Biopsy & $90(56)$ & $78(80)$ & $2(4)$ & $2(8)$ \\
\hline Excision & $70(44)$ & $19(20)$ & $51(96)$ & $23(92)$ \\
\hline \multicolumn{5}{|l|}{ Hormone receptor } \\
\hline Negative & $59(37)$ & $28(29)$ & $33(62)$ & $3(12)$ \\
\hline Positive & $99(62)$ & $68(70)$ & $20(38)$ & $22(88)$ \\
\hline Unknown & $2(1)$ & $1(1)$ & $0(0)$ & $0(0)$ \\
\hline \multicolumn{5}{|l|}{ HER2 } \\
\hline Negative & $111(69)$ & $62(64)$ & $24(45)$ & $21(84)$ \\
\hline Positive & $44(28)$ & $33(34)$ & $29(55)$ & $4(16)$ \\
\hline Unknown & $5(3)$ & $2(2)$ & $0(0)$ & $0(0)$ \\
\hline \multicolumn{5}{|l|}{ Subtype } \\
\hline HR+/HER2- & $77(48)$ & $56(58)$ & $8(15)$ & $18(72)$ \\
\hline $\mathrm{HR}+/ \mathrm{HER} 2+$ & $17(11)$ & $11(11)$ & $12(23)$ & $4(16)$ \\
\hline HR-/HER $2+$ & $25(16)$ & $22(23)$ & $17(32)$ & $0(0)$ \\
\hline $\mathrm{TN}$ & $34(21)$ & $6(6)$ & $16(30)$ & $3(12)$ \\
\hline Unknown & $7(4)$ & $2(2)$ & $0(0)$ & $0(0)$ \\
\hline \multicolumn{5}{|c|}{ Concordance of subtypes between primary and metastatic sites } \\
\hline Concordant & $106(66)$ & $69(71)$ & $40(76)$ & $20(80)$ \\
\hline Discordant & $25(16)$ & $12(12)$ & $9(17)$ & $5(20)$ \\
\hline Unknown & $29(18)$ & $16(17)$ & $4(7)$ & $0(0)$ \\
\hline TIL (\%, median) & 10 & 2 & 5 & 2 \\
\hline \multicolumn{5}{|l|}{ TLS } \\
\hline Absent & $79(49)$ & $71(74)$ & $37(70)$ & $25(100)$ \\
\hline Present & $48(30)$ & $13(13)$ & $0(0)$ & $0(0)$ \\
\hline Unknown & $33(21)$ & $13(13)$ & $16(30)$ & $0(0)$ \\
\hline
\end{tabular}

DMFI distant metastasis-free interval, $H R$ hormone receptor, TIL tumor-infiltrating lymphocyte, $T L S$ tertiary lymphoid structure, $T N$ triple-negative

lung was higher than those in primary tumors $(P<0.001)$, while metastatic tumors of the liver $(P=0.007)$ and brain $(P=0.014)$ showed lower level of tumor-infiltrating lymphocytes than primary tumors (Tables 1 and 2, Fig. 2). We also analyzed pair-wise tumor-infiltrating lymphocyte 
differences between primary tumors and corresponding metastatic sites and found the same pattern (Fig. 1b).

\section{Presence of tertiary lymphoid structure in primary and metastatic cancers}

Tertiary lymphoid structures are considered to be important sites for the initiation and/or maintenance of local and systemic anti-tumor immune responses [24]. However, tertiary lymphoid structures are scattered among tumor microenvironments, and the tertiary lymphoid structure assessments from breast cancer biopsies and corresponding surgical tissues have shown poor correlations [17]. Therefore, to examine the association between the level of tumorinfiltrating lymphocytes and the presence of tertiary lymphoid structures, we analyzed surgically excised specimens from both primary tumor and metastases and assessed the presence of tertiary lymphoid structures $(n=101)$. Primary tumors with the presence of tertiary lymphoid structures showed significantly higher tumor-infiltrating lymphocyte levels than those without tertiary lymphoid structures, across all metastatic groups (lung, median 5 vs. 2, $P=0.001$; liver, 10 vs. $2, P=0.009$; brain, 10 vs. 2 , $P<0.001$; ovary, 10 vs. $2, P=0.011)$.

Tertiary lymphoid structures were present in all primary breast cancers and the four metastatic sites at following ratios: lung, 58/103; liver, 23/52; brain, 9/32; ovary, 3/21. However, in metastases, tertiary lymphoid structures were only found in the lung (32/48) and liver (6/13) metastases (Fig. 3a and Table 2). Metastatic tumors in the lungs with tertiary lymphoid structures showed significantly higher levels of tumor-infiltrating lymphocytes than metastatic lesions without tertiary lymphoid structures (median, 25 vs. $10, P=0.001$, Fig. 3b). Metastatic tumors in the liver with tertiary lymphoid structures also showed higher levels of tumor-infiltrating lymphocytes than metastatic lesions without tertiary lymphoid structures, but the difference was not statistically significant (median 5 vs. $2, P=0.694$, Fig. $3 b$ ).

Next, we analyzed the effect of tertiary lymphoid structure differences between primary tumors and metastases. In cases of lung metastases, tumor-infiltrating lymphocyte levels in the metastasis were higher than in the matched
Fig. 2 Tumor-infiltrating lymphocytes in primary tumors and metastases. Upper panel, a case with low level of tumorinfiltrating lymphocytes in primary breast cancer and high level of tumor-infiltrating lymphocytes in lung metastasis; middle panel, a case with high level of tumor-infiltrating lymphocytes in primary breast cancer and low level of tumorinfiltrating lymphocytes in brain metastasis; lower panel, a case of primary breast cancer and corresponding liver metastasis with similar low level of tumorinfiltrating lymphocytes in both sites
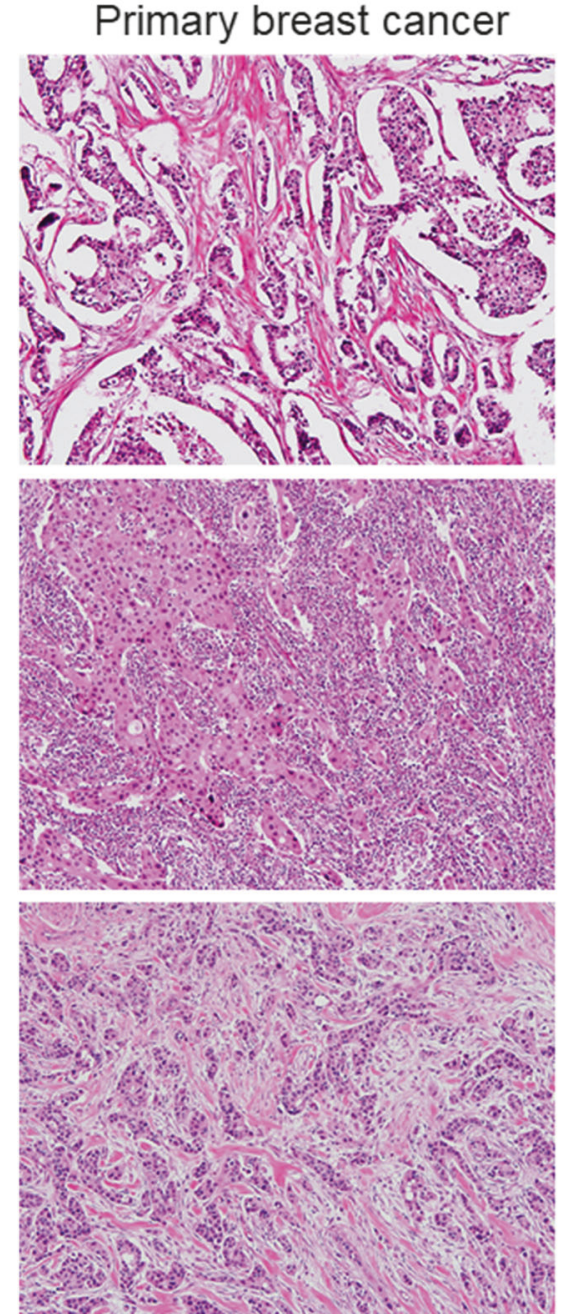
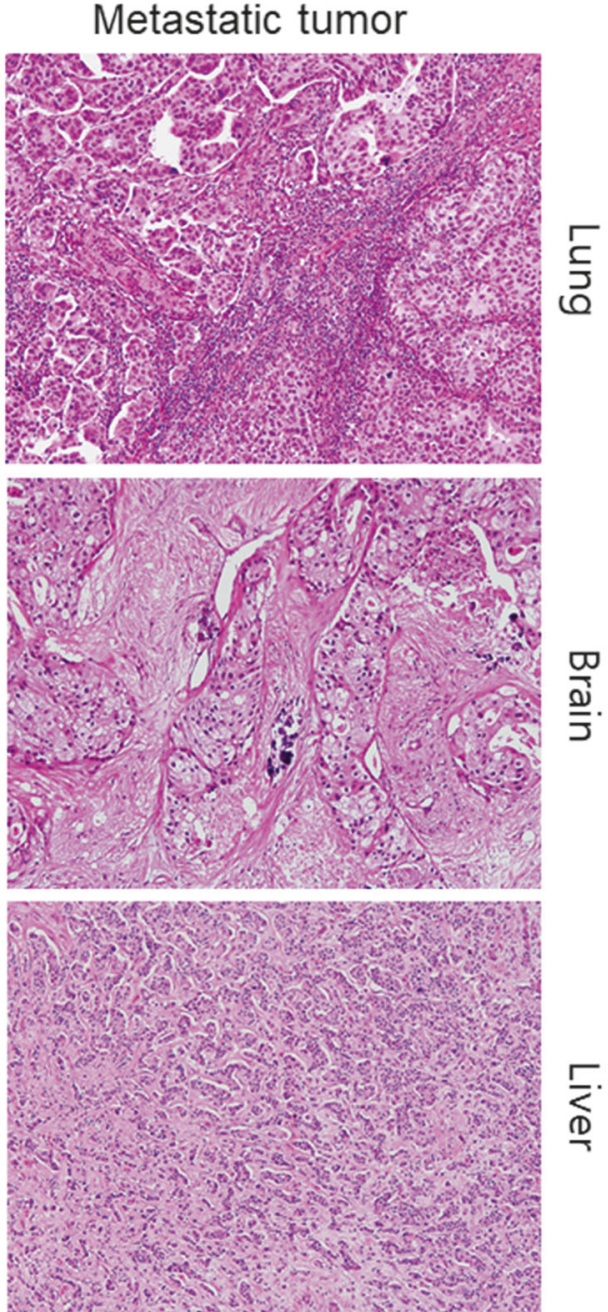

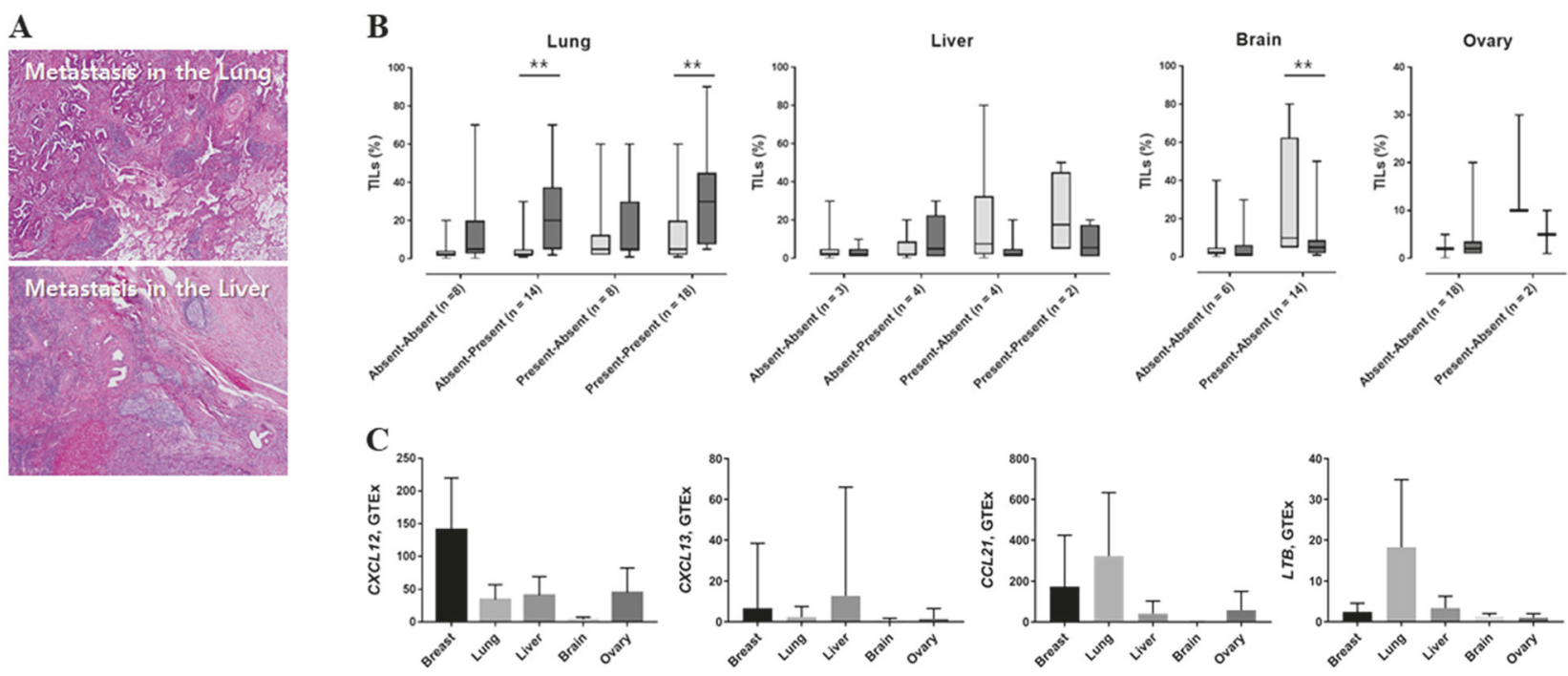
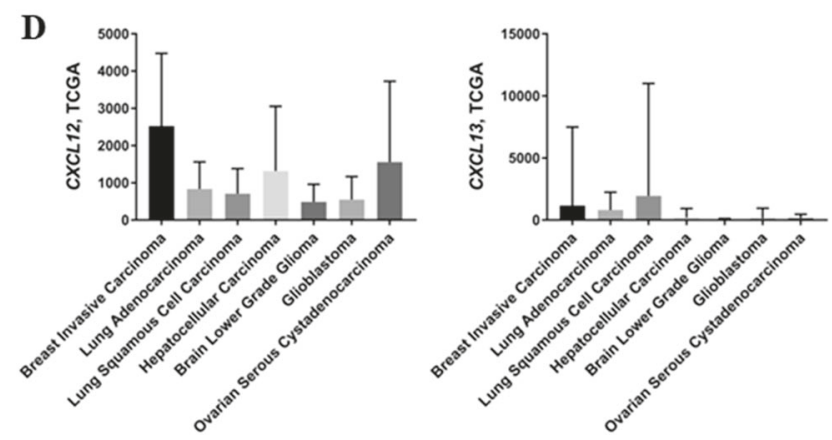

Fig. 3 Tertiary lymphoid structure. a Tertiary lymphoid structures in the lung and liver metastases (40×, hematoxylin and eosin-stained). b Comparison of tumor-infiltrating lymphocytes (tumor-infiltrating lymphocytes) in primary tumors and metastases, according to changes in tertiary lymphoid structure presence from the primary tumor to metastases (box filling color, light gray, primary tumor; dark gray, metastasis). ${ }^{*} P(<0.01$. c Comparison of CXCL12, CXCL13, CCL21, and $L T B$ gene expression (TPM, Transcripts Per Million) from the Genotype-Tissue Expression Project in the breast $(n=290)$, lung

primary tumors for all groups, except the group with the presence of tertiary lymphoid structures in primary tumors and absence in metastasis (Fig. 3b). In cases of brain metastases, the change of tertiary lymphoid structures from present to absent significantly affected the tumor-infiltrating lymphocyte levels in metastases compared with matched primary tumors $(P=0.002)$.

To compare the chemokines associated with lymphoid genesis in different organs, we analyzed CXCL12, CXCL13, CCL21, and LTB gene expression levels from the Genotype-Tissue Expression database [25]. The brain showed significantly lower expression of all four genes than the breast, lung, and liver (Fig. 3c). In The Cancer Genome Atlas data analysis, brain tumors also showed lower expression of these genes than breast, lung, and liver tumors (Fig. 3d).
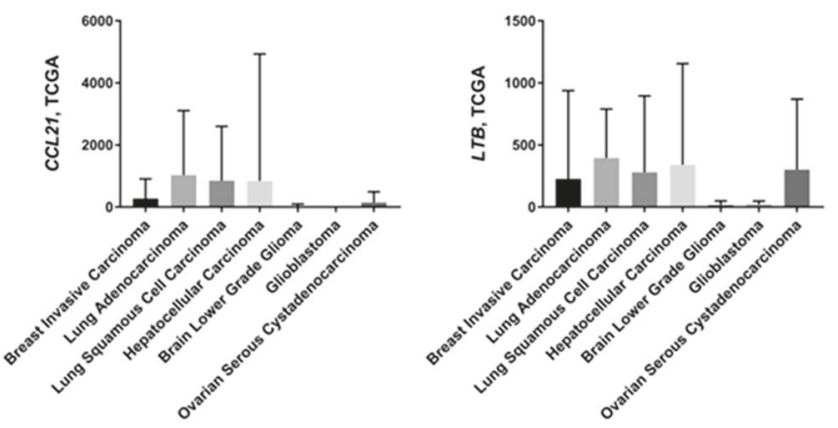

$(n=427)$, liver $(n=175)$, brain $(n=1671)$, and ovary $(n=133)$. d Comparison of CXCL12, CXCL13, CCL21, and LTB gene expression (RSEM, RNA-Seq by Expectation Maximization) from The Cancer Genome Atlas data in breast invasive carcinoma $(n=1100)$, lung adenocarcinoma $(n=517)$, lung squamous cell carcinoma $(n$ $=501)$, hepatocellular carcinoma $(n=373)$, brain low grade glioma $(n=530)$, glioblastoma $(n=167)$, and serous ovarian cystadenocarcinoma $(n=307)$

\section{The prognostic significance of tumor-infiltrating lymphocytes and tertiary lymphoid structures in metastatic sites}

Patients with lower histological grade, hormone receptor positivity in primary tumors and metastases, lower primary tumor-infiltrating lymphocyte levels, higher tumorinfiltrating lymphocyte levels in metastases, absence of tertiary lymphoid structures in the primary tumors, presence of tertiary lymphoid structures in metastases, lung metastases, a single metastatic mass, and metastatic site excision showed significantly better overall survival (Table 3). In patients with lung metastases, a lower histological grade, hormone receptor positivity in the primary tumors and metastases, higher tumor-infiltrating lymphocyte levels in metastases, and absence of tertiary lymphoid structures in 


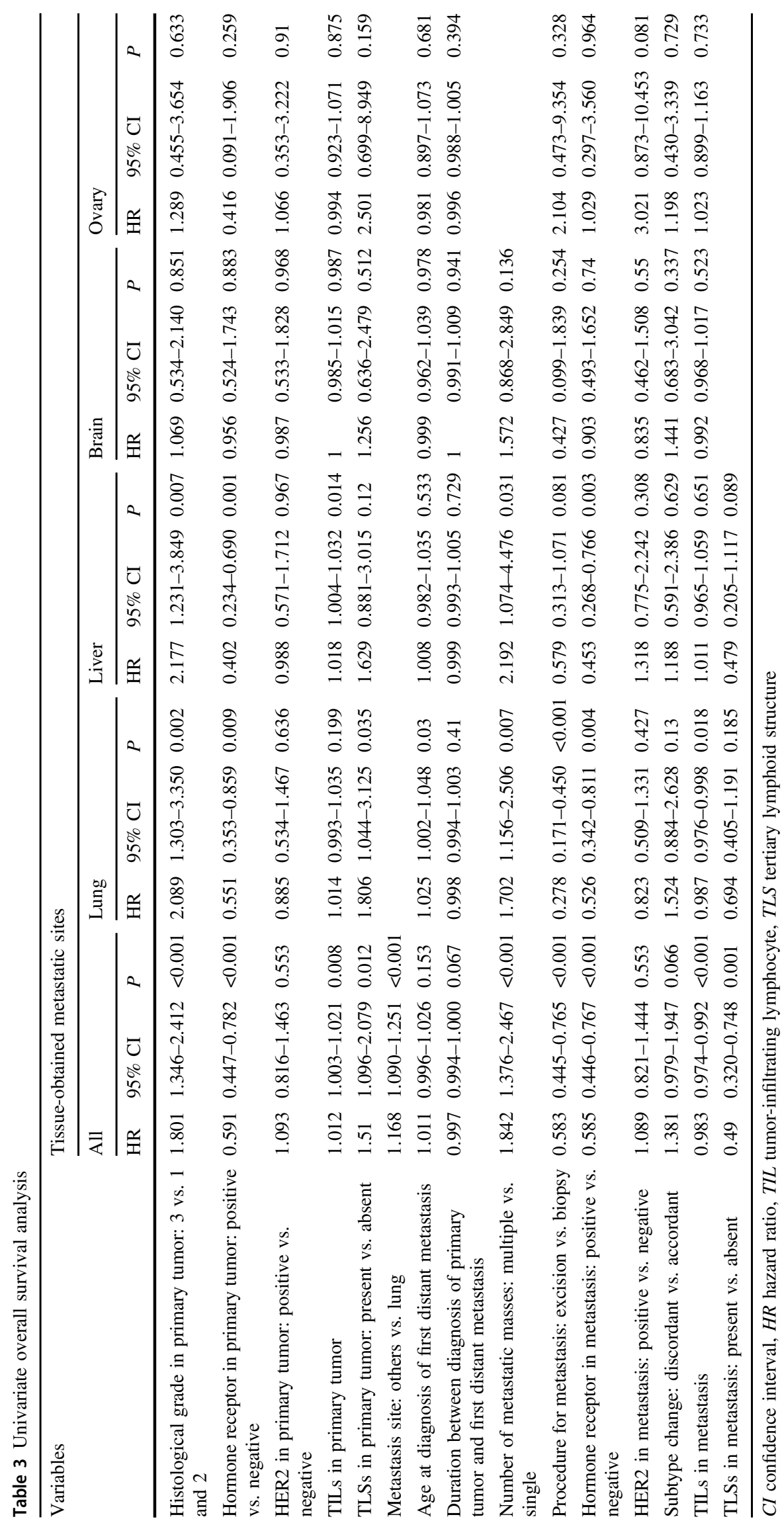


Table 4 Multivariate overall survival analysis

\begin{tabular}{|c|c|c|c|c|c|c|c|c|c|}
\hline \multirow[t]{3}{*}{ Variables } & \multicolumn{9}{|c|}{ Tissue-obtained metastatic sites } \\
\hline & \multicolumn{3}{|l|}{ All } & \multicolumn{3}{|l|}{ Lung } & \multicolumn{3}{|l|}{ Liver } \\
\hline & HR & $95 \% \mathrm{CI}$ & $P$ & HR & $95 \% \mathrm{CI}$ & $P$ & HR & $95 \% \mathrm{CI}$ & $P$ \\
\hline Histological grade in primary tumor: 3 vs. 1 and 2 & 2.021 & $1.376-2.968$ & $<0.001$ & 3.617 & $1.944-6.729$ & $<0.001$ & 3.115 & $1.564-6.201$ & 0.001 \\
\hline TILs in primary tumor & & & & & & & 1.016 & $1.001-1.031$ & 0.035 \\
\hline Metastasis site: others vs. lung & 1.197 & $1.083-1.323$ & $<0.001$ & & & & & & \\
\hline Age at diagnosis of first distant metastasis & & & & 1.032 & $1.007-1.059$ & 0.013 & & & \\
\hline Number of metastatic masses: multiple vs. single & 1.779 & $1.229-2.574$ & 0.002 & 1.903 & $1.176-3.078$ & 0.009 & & & \\
\hline Procedure for metastasis: excision vs. biopsy & & & & 0.351 & $0.191-0.642$ & 0.001 & & & \\
\hline Hormone receptor in metastasis: positive vs. negative & 0.628 & $0.425-0.927$ & 0.019 & 0.384 & $0.217-0.680$ & 0.001 & & & \\
\hline
\end{tabular}

CI confidence interval, $H R$ hazard ratio, $T I L$ tumor-infiltrating lymphocyte, $T L S$ tertiary lymphoid structure

the primary tumors showed significantly better clinical outcomes. In patients with liver metastases, histological grade, hormone receptor status in the primary tumors and metastases, tumor-infiltrating lymphocyte levels in the primary tumors, and the number of metastatic masses were associated with overall survival. In patients with brain or ovary metastases, no variables were prognosticators.

In multivariate analysis, histological grade, metastatic site, number of metastatic masses, and hormone receptor status of metastases were independent prognostic factors for overall survival (Table 4). In subgroup analysis of lung cancer metastasis, histological grade, age at diagnosis of first distant metastasis, number of metastatic masses, procedure for metastasis, and hormone receptor status of the metastases were independent prognostic factors. In the liver metastasis group, histological subtype and the level of tumor-infiltrating lymphocytes in the primary tumor were independent prognostic factors.

\section{Lung metastasis tumor-infiltrating lymphocyte level was higher in excised metastatic tumor specimens than in biopsy only samples}

In lung metastasis, patients with excised metastatic lesions showed better survival outcomes than those with only lung biopsy. The tumor-infiltrating lymphocyte levels were significantly higher in cases with excised metastases than in those with biopsy only for lung metastases (median, 20 vs. 5, $P<0.001)$. To exclude the possibility of discrepancy between tumor-infiltrating lymphocyte levels in biopsy and excision specimens, we compared tumor-infiltrating lymphocyte levels between biopsies and excisions of the same metastatic lesion in 12 patients with lung metastases. The tumor-infiltrating lymphocytes in biopsies and excisions of the same metastatic lesion correlated with each other ( $\rho=0.773, P=0.003$ ), and tumor-infiltrating lymphocytes did not differ between biopsies and excisions (median, 12.5 vs. $20, P=0.281)$.

\section{Discussion}

Comparisons of tumor-infiltrating lymphocytes between primary and metastatic breast cancers have been previously published; however, these studies analyzed either a small number of cases or only one metastatic organ [26-29]. Our study aims to evaluate tertiary lymphoid structures in breast cancer metastatic sites and tumor-infiltrating lymphocytes, using the largest number of metastatic breast cancer cases. Interestingly, we found higher level of tumor-infiltrating lymphocytes in lung metastases than in metastases of other organs, regardless of breast cancer subtypes (Fig. 1 and Table 2). In addition, tumor-infiltrating lymphocyte levels in lung metastases were significantly higher than those in matched primary tumors. On the other hand, tumorinfiltrating lymphocyte levels decreased in liver and brain metastases. Similar to our results, it has been reported that the number of $\mathrm{CD} 4+$ and $\mathrm{CD} 8+\mathrm{T}$ cells significantly decreased in brain $(n=21$ and 46) and liver $(n=11)$ metastases, compared with the primary breast cancer [27, 28]. A small study has also reported that despite the increased tumor-infiltrating lymphocyte levels in lung metastases $(n=7)$, tumor-infiltrating lymphocyte levels in metastatic lesions $(n=26)$ generally decreased, compared with the corresponding primary breast cancer [29]. Additional reports have supported our results. Mansfield et al. found less tumor-infiltrating lymphocytes in brain metastases than the primary lung cancer $(P<0.0001)$ and Kluger et al. reported lower CD3-positive $\mathrm{T}$ cells in cerebral metastases of melanoma than in extracerebral metastases $(P=0.010)$ [30, 31]. Since lung is an organ with constant exposure of pathogens/allergens, early response to these stimuli is essential for survival [32]. Respiratory epithelium can produce various cytokines, and antigen-presenting cells such as dendritic cells and macrophages reside in airways. These specific features of lung might cause significantly higher level of tumor-infiltrating lymphocytes in lung metastases than in other metastatic sites. 
The prognostic significance of tertiary lymphoid structures was evaluated in various primary cancers, including melanoma, breast, gastric, pancreatic, non-small cell lung, oral squamous cell, and renal cell cancer, hepatocellular carcinoma, and cholangiocarcinoma [18]. Presence of tertiary lymphoid structures in metastatectomy lesions has been reported in pulmonary metastasis of colorectal cancer [33]. Seventy-nine percentage of pulmonary metastasis showed presence of tertiary lymphoid structures. In pairwise analysis, metastases (22/28) were more frequently tertiary lymphoid structure-positive than matched primary colorectal cancers $(2 / 28, P<0.001)$. In addition, the levels of $\mathrm{CD}^{+}, \mathrm{CD}^{+}$, and $\mathrm{CD} 45 \mathrm{RO}^{+} \mathrm{T}$ cells were significantly higher in pulmonary metastases. Although we found higher levels of tumor-infiltrating lymphocytes in lung metastases than in corresponding primary breast cancers, tertiary lymphoid structure positivity was not different between primary tumors (26/48) and metastases $(32 / 48, P=0.286)$. It seems that the formation of tertiary lymphoid structure in metastatic sites might be controlled by several factors, such as type of primary cancer and microenvironment. Hence, further evaluation of tumor-infiltrating lymphocytes and tertiary lymphoid structures in lung metastases from various primary organs is needed, to clarify the factors that affect the formation of tertiary lymphoid structures in lung metastases.

There were no tertiary lymphoid structures in the brain and ovary metastases of our study population. Based on CXCL13 gene expression levels, which are significantly associated with the presence of tertiary lymphoid structures, in The Cancer Genome Atlas data of various tumor types, we could assume that primary brain tumors also do not frequently have tertiary lymphoid structures (Fig. 2d, from www.cbioportal.org) [18, 22]. Brain tumor metastases consist mostly of HER2-positive or triple-negative tumors $(85 \%)$, which are immunogenic subtypes with higher tumor-infiltrating lymphocyte levels in the primary breast cancers [23]. Although breast cancer subtype influences the infiltration of $\mathrm{T}$ cells into primary sites, it seems that the level of tumor-infiltrating lymphocytes in metastatic brain lesions is affected by the presence of tertiary lymphoid structures, considering the abrupt decrease of tumorinfiltrating lymphocytes and absence of tertiary lymphoid structure in metastatic brain lesions, compared to primary tumors (Figs. 1, 2). Several studies have evaluated tumorinfiltrating lymphocytes in brain tumors and found infiltration of various immune cells with relatively low density [34]. However, we currently lack an understanding of immune interactions in brain tumors. On the other hand, the absence of tertiary lymphoid structures in ovarian metastasis might be associated with the low immunogenic potential of hormone receptor-positive tumors, which is the predominant subtype of primary tumors with ovarian metastasis.

We found significant association between the presence of tertiary lymphoid structures and tumor-infiltrating lymphocyte levels and prognostic significance of tumor-infiltrating lymphocytes in metastases (Fig. 2, Tables 3 and 4). It has been suggested that tertiary lymphoid structure modulation could effectively increase tumor-infiltrating lymphocyte levels and subsequently improve clinical outcomes. In addition, Sautès-Fridman et al. suggested targeting the molecules that are involved in lymphoid organogenesis, such as LT $\beta \mathrm{R}$ and IL-7 receptor signaling pathways and CXCL13/CXCR5 and IL-21 chemokine and cytokine networks [18]. However, these methods might not be effective in organs where it is intrinsically difficult to generate tertiary lymphoid structures, such as the brain. Therefore, specialized approaches may be required for each metastatic organ.

Buisseret et al. assessed tumor-infiltrating lymphocytes in breast cancer biopsies and surgical samples and found strong correlations between two specimens $\left(R^{2}=0.74, P<\right.$ 0.001 ) [17]. In the present study, we also found significant positive association between tumor-infiltrating lymphocytes of biopsies and excisions from the same breast cancer metastatic lesion (Tables 3 and 4). The significantly higher tumor-infiltrating lymphocyte levels in cases with lung metastasis excision, rather than biopsy alone, suggests that an increased anti-tumor response in some tumors with lung metastases could control the metastatic spread of breast cancer and make surgical resection of the metastatic lesion possible.

Although we evaluated the largest number of cases of metastatic breast cancer in terms of tumor-infiltrating lymphocytes, our retrospective study design and small sample size are limitations of our study.

In conclusion, this study evaluated the largest number of cases for metastatic breast cancer in terms of tumorinfiltrating lymphocytes and tertiary lymphoid structures. We showed that metastatic breast tumors in the lung had more tumor-infiltrating lymphocytes than those at other sites and matched primary tumors. In addition, we found that the presence of tertiary lymphoid structures in metastatic sites are a critical factor for tumor-infiltrating lymphocyte levels. We also confirmed that the tumorinfiltrating lymphocyte levels in metastatic lesions are prognostic factors for metastatic breast cancer.

Acknowledgements This study was supported by the Korean Health Technology R\&D Project, Ministry of Health \& Welfare (HI15C0708 and HI17C0337) and Basic Science Research Programs through the National Research Foundation of Korea (NRF) funded by the Ministry of Science, ICT \& Future Planning, Republic of Korea (NRF2017R1D1A1B03027977). 


\section{Compliance with ethical standards}

Conflict of interest The authors declare that they have no conflict of interest.

\section{References}

1. Hendry S, Salgado R, Gevaert T,Russell PA, John T, Thapa B, et al. Assessing tumor-infiltrating lymphocytes in solid tumors: a practical review for pathologists and proposal for a standardized method from the International Immunooncology Biomarkers Working Group: part 1: assessing the host immune response, tumor-infiltrating lymphocytes in invasive breast carcinoma and ductal carcinoma in situ, metastatic tumor deposits and areas for further research. Adv Anat Pathol. 2017;24:235-51.

2. Lee HJ, Kim JY, Park IA, Song IH, Yu JH, Ahn JH, et al. Prognostic significance of tumor-infiltrating lymphocytes and the tertiary lymphoid structures in HER2-positive breast cancer treated with adjuvant trastuzumab. Am J Clin Pathol. 2015;144:278-88.

3. Lee HJ, Park IA, Song IH, Shin SJ, Kim JY, Yu JH, et al. Tertiary lymphoid structures: prognostic significance and relationship with tumour-infiltrating lymphocytes in triple-negative breast cancer. J Clin Pathol. 2016;69:422-30.

4. Song IH, Heo SH, Bang WS, Park HS, Park IA, Kim YA, et al. Predictive value of tertiary lymphoid structures assessed by high endothelial venule counts in the neoadjuvant setting of triplenegative breast cancer. Cancer Res Treat. 2017;49:399-407.

5. Remark R, Alifano M, Cremer I, Lupo A, Dieu-Nosjean MC, Riquet M, et al. Characteristics and clinical impacts of the immune environments in colorectal and renal cell carcinoma lung metastases: influence of tumor origin. Clin Cancer Res. 2013;19:4079-91.

6. Cipponi A, Mercier M, Seremet T, Baurain JF, Theate I, van den Oord J, et al. Neogenesis of lymphoid structures and antibody responses occur in human melanoma metastases. Cancer Res. 2012;72:3997-4007.

7. Salgado R, Denkert C, Demaria S, Sirtaine N, Klauschen F, Pruneri G, et al. The evaluation of tumor-infiltrating lymphocytes (tumor-infiltrating lymphocytes) in breast cancer: recommendations by an International tumor-infiltrating lymphocytes Working Group 2014. Ann Oncol. 2015;26:259-71.

8. Denkert C, Loibl S, Noske A, Roller M, Muller BM, Komor M, et al. Tumor-associated lymphocytes as an independent predictor of response to neoadjuvant chemotherapy in breast cancer. J Clin Oncol. 2010;28:105-13.

9. Mohme M, Schliffke S, Maire CL, Runger A, Glau L, Mende KC, et al. Immunophenotyping of newly diagnosed and recurrent glioblastoma defines distinct immune exhaustion profiles in peripheral and tumor-infiltrating lymphocytes. Clin Cancer Res. 2018. Feb 14. doi: 10.1158/1078-0432.CCR-17-2617. [Epub ahead of print]

10. Welters MJP, Ma W, Santegoets S, Goedemans R, Ehsan I, Jordanova ES, et al. Intratumoral HPV16-specific T cells constitute a type I-oriented tumor microenvironment to improve survival in HPV16-driven oropharyngeal cancer. Clin Cancer Res. 2018;24:634-47.

11. Zheng C, Zheng L, Yoo JK, Guo H, Zhang Y, Guo X, et al. Landscape of infiltrating $\mathrm{T}$ cells in liver cancer revealed by singlecell sequencing. Cell. 2017;169:1342-56 e1316.

12. Denkert C, Wienert S, Poterie A, Loibl S, Budczies J, Badve S, et al. Standardized evaluation of tumor-infiltrating lymphocytes in breast cancer: results of the ring studies of the international immuno-oncology biomarker working group. Mod Pathol. 2016;29:1155-64.

13. Luen SJ, Salgado R, Fox S, Savas P, Eng-Wong J, Clark E, et al. Tumour-infiltrating lymphocytes in advanced HER2-positive breast cancer treated with pertuzumab or placebo in addition to trastuzumab and docetaxel: a retrospective analysis of the CLEOPATRA study. Lancet Oncol. 2017;18:52-62.

14. Loi S, Sirtaine N, Piette F, Salgado R, Viale G, Van Eenoo F, et al. Prognostic and predictive value of tumor-infiltrating lymphocytes in a phase III randomized adjuvant breast cancer trial in node-positive breast cancer comparing the addition of docetaxel to doxorubicin with doxorubicin-based chemotherapy: BIG 02-98. J Clin Oncol. 2013;31:860-7.

15. Adams S, Gray RJ, Demaria S, Goldstein L, Perez EA, Shulman $\mathrm{LN}$, et al. Prognostic value of tumor-infiltrating lymphocytes in triple-negative breast cancers from two phase III randomized adjuvant breast cancer trials: ECOG 2197 and ECOG 1199. J Clin Oncol. 2014;32:2959-66.

16. Dieci MV, Mathieu MC, Guarneri V, Conte P, Delaloge S, Andre F, et al. Prognostic and predictive value of tumor-infiltrating lymphocytes in two phase III randomized adjuvant breast cancer trials. Ann Oncol. 2015;26:1698-704.

17. Buisseret L, Desmedt C, Garaud S, Fornili M, Wang X, Van den Eyden G, et al. Reliability of tumor-infiltrating lymphocyte and tertiary lymphoid structure assessment in human breast cancer. Mod Pathol. 2017;30:1204-12.

18. Sautes-Fridman C, Lawand M, Giraldo NA, Kaplon H, Germain C, Fridman WH, et al. Tertiary lymphoid structures in cancers: prognostic value, regulation, and manipulation for therapeutic intervention. Front Immunol. 2016;7:407.

19. Harvey JM, Clark GM, Osborne CK, Allred DC. Estrogen receptor status by immunohistochemistry is superior to the ligandbinding assay for predicting response to adjuvant endocrine therapy in breast cancer. J Clin Oncol. 1999;17:1474-81.

20. Wolff AC, Hammond ME, Hicks DG, Dowsett M, McShane LM, Allison $\mathrm{KH}$, et al. Recommendations for human epidermal growth factor receptor 2 testing in breast cancer: American Society of Clinical Oncology/College of American Pathologists clinical practice guideline update. Arch Pathol Lab Med. 2014;138:241-56.

21. Carithers LJ, Ardlie K, Barcus M, Branton PA, Britton A, Buia $\mathrm{SA}$, et al. A novel approach to high-quality postmortem tissue procurement: the GTEx project. Biopreserv Biobank. 2015;13:311-9.

22. Gao J, Aksoy BA, Dogrusoz U, Dresdner G, Gross B, Sumer SO, et al. Integrative analysis of complex cancer genomics and clinical profiles using the cBioPortal. Sci Signal. 2013;6:pl1.

23. Stanton SE, Disis ML. Clinical significance of tumor-infiltrating lymphocytes in breast cancer. J Immunother Cancer. 2016;4:59.

24. Dieu-Nosjean MC, Giraldo NA, Kaplon H, Germain C, Fridman WH, Sautes-Fridman C. Tertiary lymphoid structures, drivers of the anti-tumor responses in human cancers. Immunol Rev. 2016;271:260-75.

25. Silina K, Soltermann A, Movahedian Attar F, Casanova R, Uckeley ZM, Thut $\mathrm{H}$, et al. Germinal centers determine the prognostic relevance of tertiary lymphoid structures and are impaired by corticosteroids in lung squamous cell carcinoma. Cancer Res. 2018;78:1308-20.

26. Ogiya R, Niikura N, Kumaki N, Bianchini G, Kitano S, Iwamoto $\mathrm{T}$, et al. Comparison of tumor-infiltrating lymphocytes between primary and metastatic tumors in breast cancer patients. Cancer Sci. 2016;107:1730-5.

27. Ogiya R, Niikura N, Kumaki N, Yasojima H, Iwasa T, Kanbayashi $\mathrm{C}$, et al. Comparison of immune microenvironments between primary tumors and brain metastases in patients with breast cancer. Oncotarget. 2017;8:103671-81. 
28. Sobottka B, Pestalozzi B, Fink D, Moch H, Varga Z. Similar lymphocytic infiltration pattern in primary breast cancer and their corresponding distant metastases. Oncoimmunology. 2016;5: e1153208.

29. Cimino-Mathews A, Thompson E, Taube JM, Ye X, Lu Y, Meeker A, et al. PD-L1 (B7-H1) expression and the immune tumor microenvironment in primary and metastatic breast carcinomas. Hum Pathol. 2016;47:52-63.

30. Mansfield AS, Aubry MC, Moser JC, Harrington SM, Dronca RS, Park SS, et al. Temporal and spatial discordance of programmed cell death-ligand 1 expression and lymphocyte tumor infiltration between paired primary lesions and brain metastases in lung cancer. Ann Oncol. 2016;27:1953-8.
31. Kluger HM, Zito CR, Barr ML, Baine MK, Chiang VL, Sznol M, et al. Characterization of PD-L1 expression and associated T-cell infiltrates in metastatic melanoma samples from variable anatomic sites. Clin Cancer Res. 2015;21:3052-60.

32. Moldoveanu B, Otmishi P, Jani P, Walker J, Sarmiento X, Guardiola $\mathbf{J}$, et al. Inflammatory mechanisms in the lung. $\mathbf{J}$ Inflamm Res. 2009;2:1-11.

33. Schweiger T, Berghoff AS, Glogner C, Glueck O, Rajky O, Traxler D, et al. Tumor-infiltrating lymphocyte subsets and tertiary lymphoid structures in pulmonary metastases from colorectal cancer. Clin Exp Metastas. 2016;33:727-39.

34. Bienkowski M, Preusser M. Prognostic role of tumour-infiltrating inflammatory cells in brain tumours: literature review. Curr Opin Neurol. 2015;28:647-58. 